\title{
Changes in inflammatory factors and prognosis of patients complicated with non-alcoholic fatty liver disease undergoing coronary artery bypass grafting
}

\author{
LEI WANG $^{1 *}$, YUANMING LI $^{1 *}$ and XIAOJIN GONG ${ }^{2}$ \\ Departments of ${ }^{1}$ Surgery, Ward 3 and ${ }^{2}$ Pathology, The Second Affiliated Hospital of \\ Xinjiang Medical University, Urumqi, Xinjiang 830028, P.R. China
}

Received July 19, 2017; Accepted November 2, 2017

DOI: $10.3892 /$ etm.2017.5476

\begin{abstract}
This study was designed to assess the relationship between changes in peripheral inflammatory factors of patients complicated with non-alcoholic fatty liver disease (NAFLD) undergoing coronary artery bypass grafting (CABG) before and after operation, and their prognosis. A total of 68 patients with stable angina pectoris treated in Xinjiang Hospital who underwent CABG at some point between August of 2013 and August of 2015 were enrolled in the study, and divided into the NAFLD group $(n=31)$ and the non-NAFLD group $(n=37)$ according to the presence of the condition or its absence. Peripheral blood was drawn from the patients before and at $24 \mathrm{~h}$ and 1 month after the operation, and the expression levels of high-sensitivity C-reactive protein (hsCRP), soluble CD40 ligand (sCD40L), intercellular adhesion molecule-1 (ICAM-1) and matrix metalloproteinase-9 (MMP-9) were measured via standard enzyme-linked immunosorbent assay. Our results showed the expression levels of hsCRP and sCD40L of patients in both groups reached a peak $24 \mathrm{~h}$ after operation; and there were statistically significant changes compared with the levels before the operation and at 1 month after operation $(\mathrm{p}<0.01)$. However, there were no statistically significant differences in the expression levels between the two groups ( $p>0.05)$. The expression levels of ICAM-1 at each time-point in both groups were increased after the operation, but the changes were not statistically significant $(p>0.05)$. The expression levels of MMP-9 increased after the operation, and the levels at 1 month after operation were significantly higher
\end{abstract}

Correspondence to: Dr Lei Wang, Department of Surgery, Ward 3, The Second Affiliated Hospital of Xinjiang Medical University, 1284 Qidaowan Road, Shuimogou, Urumqi, Xinjiang 830028, P.R. China

E-mail: wangleixjyx@163.com

${ }^{*}$ Contributed equally

Key words: non-alcoholic fatty liver disease, coronary artery bypass grafting, matrix metalloproteinase- 9 , prognosis than those before operation and at $24 \mathrm{~h}$ after the operation $(\mathrm{p}<0.01)$. Importantly, the expression levels of MMP-9 of patients in the NAFLD group at 1 month after operation were significantly higher than those of patients in the non-NAFLD group at the same time, and the differences were statistically significant $(\mathrm{p}<0.01)$. Finally, logistic regression analysis showed that the expression level of MMP-9 was an important influencing factor for cardiovascular events after CABG $(\mathrm{OR}=1.182, \mathrm{p}<0.05)$. Based on our findings, the expression levels of inflammatory factors in peripheral blood in patients complicated with NAFLD undergoing CABG differ from those who are not complicated, and the MMP-9 levels may be closely related to the prognosis of patients complicated with NAFLD.

\section{Introduction}

With the continuous improvement of living standards in China, the incidence of non-alcoholic fatty liver disease (NAFLD), a disease previously almost exclusive of Western countries, has been increasing year by year, and it has become one of the most common liver diseases affecting human health $(1,2)$. Studies have shown that NAFLD can lead to metabolic disorders and diseases, which are closely related to hypertension and coronary heart disease (3). Cardiac bypass surgery and coronary stent implantation are common effective surgical treatments for coronary heart disease. But a series of adverse cardiovascular events after surgery still seriously threaten the life and health of those patients. Studies have shown that the bypass surgery induces the production and release of inflammatory factors in patients with coronary heart disease, affecting the prognosis of patients with coronary heart disease after surgery (4-6). At present, there are few studies on the relationship between the changes in inflammatory factors and the prognosis of coronary heart disease patients complicated with NAFLD after bypass surgery. In this study, the changes in expressions levels of inflammatory factors in peripheral blood of coronary heart disease patients complicated with NAFLD at different time-points before and after bypass surgery were investigated and their relationship to the patient prognosis were assessed, in order to provide a theoretical basis for clarifying mechanisms at play. 


\section{Patients and methods}

Patients. The subjects in this study were a total of 68 patients were admitted to Xinjiang Hospital due to stable angina pectoris from August 2013 to August 2015 scheduled to undergo coronary artery bypass grafting $(\mathrm{CABG})$. There were 30 males and 38 females aged 53-87 years. Diagnostic criteria for stable angina pectoris included the fact that the conditions of onset did not change within 1-3 months, the daily and weekly onset frequencies were roughly similar, the fatigue and degree of pain were stable, and the nature and site of pain were the same in every attack. Diagnostic criteria for NAFLD included histological changes in liver biopsy conformed to the pathological or imaging criteria of fatty liver disease, and the fact that patients had no alcohol drinking history or an alcohol consumption per week of $<140 \mathrm{~g}$; and other fatty liver-specific diseases caused by viral hepatitis or drug-induced hepatitis had been ruled out. A total of 68 patients undergoing CABG were divided into the NAFLD and the non-NAFLD control group. Other consumptive diseases were excluded in all patients enrolled and they all signed informed consent forms. All patients had complete clinical and pathological data and completed the treatment regimen. The patients were followedup for 6 months after the procedure and the major adverse cardiac events were recorded, including death, myocardial infarction and angina pectoris. The study was approved by the Ethics Committee of the Second Affiliated Hospital of Xinjiang Medical University.

$C A B G$. All the patients enrolled received routine treatment for coronary heart disease, they all took a sufficient dose of enteric-coated aspirin and clopidogrel before the operation and the same dose of unfractionated heparin during the procedure, and they all underwent the multi-position projection after CABG to ensure the procedure had been successful.

Collection and storage of samples. Peripheral venous blood $(5 \mathrm{ml})$ were drawn from patients before and at $24 \mathrm{~h}$ and 1 month after the operation. After natural coagulation, the samples were centrifuged at $1,700 \mathrm{xg}$ for $10 \mathrm{~min}$. The serum was separated, quickly frozen, and stored in the freezer at $-80^{\circ} \mathrm{C}$ for subsequent processing.

Instruments and reagents. Innova-3100 full digital cardiovascular angiographic system (Thermo Fisher Scientific, Waltham, MA, USA).

Detection of high-sensitivity C-reactive protein (hsCRP), soluble CD40 ligand (sCD40L), intercellular adhesion molecule-1 (ICAM-1) and matrix metalloproteinase-9 (MMP-9) inperipheralbloodbyenzyme-linked immunosorbent assay (ELISA). Serum samples obtained from the patients were diluted 100 times in preparation for conventional ELISA tests. Standard and diluted serum samples $(10 \mu \mathrm{l})$ were added onto each labeled reaction plate according to the instructions of the high-sensitivity hsCRP detection kit and the sCD40L detection kit (both from R\&D Systems, Inc., Minneapolis, MN, USA), the ICAM-1 detection kit or the MMP-9 detection kit (both from Invitrogen; Thermo Fisher Scientific, Inc., Waltham, MA, USA). Briefly, $100 \mu 1$ conjugate reagent were
Table I. Comparisons of general data of patients in the two groups before operation (mean \pm standard deviation).

\begin{tabular}{lccc}
\hline Index & $\begin{array}{c}\text { NAFLD } \\
\text { group }\end{array}$ & $\begin{array}{c}\text { Non-NAFLD } \\
\text { group }\end{array}$ & P-value \\
\hline Sex (male/female) & $14 / 17$ & $16 / 21$ & 0.382 \\
Age (years) & $65.3 \pm 8.9$ & $67.8 \pm 10.6$ & 0.295 \\
History of smoking (\%) & $45.6 \%$ & $42.7 \%$ & 0.423 \\
Hypertension (\%) & $77.4 \%$ & $67.6 \%$ & 0.076 \\
Aspirin & $93.5 \%$ & $94.6 \%$ & 0.589 \\
ACEI drugs & $51.6 \%$ & $51.3 \%$ & 0.657 \\
Statins & $96.8 \%$ & $94.6 \%$ & 0.569 \\
ALT (U/l) & $69.32 \pm 11.26$ & $25.65 \pm 7.52$ & $0.008^{\mathrm{a}}$ \\
\hline
\end{tabular}

Compared with non-NAFLD group, ${ }^{\mathrm{a}} \mathrm{p}<0.01$. NAFLD, non-alcoholic fatty liver disease; ALT, alanine aminotransferase.

added into each well, shaken slightly for $30 \mathrm{sec}$ and incubated for 60 to $90 \mathrm{~min}$ at room temperature. The liquid in the reaction plate was shaken dry and phosphate-buffered saline (PBS; self-prepared by our laboratory) was added to wash the reaction plate 5 times. Horseradish peroxidase (HRP) was added and shaken slightly for $30 \mathrm{sec}$ and incubated for $30 \mathrm{~min}$ at room temperature. The liquid in the reaction plate was shaken dry again, and PBS was added to wash the reaction plate 5 times. Then tetramethylbenzidine (TMB) developing solution was added for incubation at room temperature for $20 \mathrm{~min}$. Finally, $100 \mu \mathrm{l}$ of stop solution were added and shaken slightly for $30 \mathrm{sec}$, and the absorbance values of samples were measured using the microplate reader $(450 \mathrm{~nm})$. The contents of hsCRP, sCD40L, ICAM-1 and MMP-9 in samples in each group were calculated.

Statistical analysis. The data in this study were presented as mean \pm standard deviation. SPSS 19.0 software (SPSS, Inc., Chicago, IL, USA) was used for data analysis. t-test was used for intergroup comparisons, and analysis of variance was used for comparisons among groups. The homogeneity test of variance was performed and logistic regression analysis was used for the relationship between levels of inflammatory factors and the presence of postoperative adverse cardiovascular events. $\mathrm{P}<0.05$ was considered to indicate a statistically significant difference.

\section{Results}

General data analysis. The clinicopathology data and treatment plans of patients before the CABG procedure were summarized. The results on Table I showed that there were no statistically significant differences in terms of age, sex, smoking history or preventive drug use between the patients in the NAFLD and the non-NAFLD groups $(\mathrm{p}>0.05)$. Average alanine aminotransferase (ALT) levels in the NAFLD group were significantly higher than those in the non-NAFLD group, and the difference was statistically significant $(\mathrm{p}<0.01)$.

Expression level of hSCRP in peripheral blood. The expression level of hsCRP in peripheral blood of patients was 
Table II. Average hsCRP expression levels in peripheral blood of patients in the two groups.

\begin{tabular}{lcc}
\hline & \multicolumn{2}{c}{$\mathrm{hsCRP}(\mathrm{ng} / \mathrm{ml})$} \\
\cline { 2 - 3 } Detection time & $\begin{array}{c}\text { NAFLD } \\
\text { group }\end{array}$ & $\begin{array}{c}\text { Non-NAFLD } \\
\text { group }\end{array}$ \\
\hline Before operation & $8.76 \pm 4.27$ & $8.25 \pm 3.82$ \\
24 h after operation & $16.27 \pm 5.52^{\mathrm{a}}$ & $15.96 \pm 6.26^{\mathrm{a}}$ \\
1 month after operation & $9.27 \pm 5.13$ & $9.02 \pm 4.95$ \\
\hline
\end{tabular}

Compared with non-NAFLD group at each time-point, ${ }^{\mathrm{a}} \mathrm{p}<0.01$. hsCRP, high-sensitivity C-reactive protein; NAFLD, non-alcoholic fatty liver disease.

Table III. sCD40L expression levels in peripheral blood of patients in the two groups.

\begin{tabular}{lcc}
\hline & \multicolumn{2}{c}{$\mathrm{sCD} 40 \mathrm{~L}(\mathrm{ng} / \mathrm{ml})$} \\
\cline { 2 - 3 } Detection time & $\begin{array}{c}\text { NAFLD } \\
\text { group }\end{array}$ & $\begin{array}{c}\text { Non-NAFLD } \\
\text { group }\end{array}$ \\
\hline Before operation & $4.86 \pm 1.76$ & $4.51 \pm 1.32$ \\
24 h after operation & $9.25 \pm 2.01^{\mathrm{a}}$ & $8.97 \pm 2.38^{\mathrm{a}}$ \\
1 month after operation & $5.29 \pm 2.48$ & $4.89 \pm 1.96$ \\
\hline
\end{tabular}

Compared with non-NAFLD group at each time-point, ${ }^{\mathrm{a}} \mathrm{p}<0.01$. sCD40L, soluble CD40 ligand; NAFLD, non-alcoholic fatty liver disease.

Table IV. ICAM-1 expression levels in peripheral blood of patients in the two groups.

\begin{tabular}{lcc}
\hline & \multicolumn{2}{c}{ ICAM-1 (ng/ml) } \\
\cline { 2 - 3 } Detection time & $\begin{array}{c}\text { NAFLD } \\
\text { group }\end{array}$ & $\begin{array}{c}\text { Non-NAFLD } \\
\text { group }\end{array}$ \\
\hline Before operation & $12.06 \pm 4.23$ & $11.95 \pm 4.86$ \\
24 h after operation & $13.67 \pm 5.95$ & $13.25 \pm 6.03$ \\
1 month after operation & $11.96 \pm 5.26$ & $11.83 \pm 4.77$ \\
\hline
\end{tabular}

ICAM-1, intercellular adhesion molecule-1; NAFLD, non-alcoholic fatty liver disease.

detected using an hsCRP kit. The results on Table II and Fig. 1 show that the expression levels of hsCRP in the NAFLD and non-NAFLD groups were significantly higher at $24 \mathrm{~h}$ after the $\mathrm{CABG}$ operation than before or one month after the procedure in each group $(\mathrm{p}<0.01)$. However, there were no statistically significant differences in the expression levels of hsCRP before and at 1 month after the operation ( $p>0.05$ ); and also there were no statistically significant differences in the expression levels of hsCRP between the NAFLD and non-NAFLD groups ( $\mathrm{p}>0.05)$.

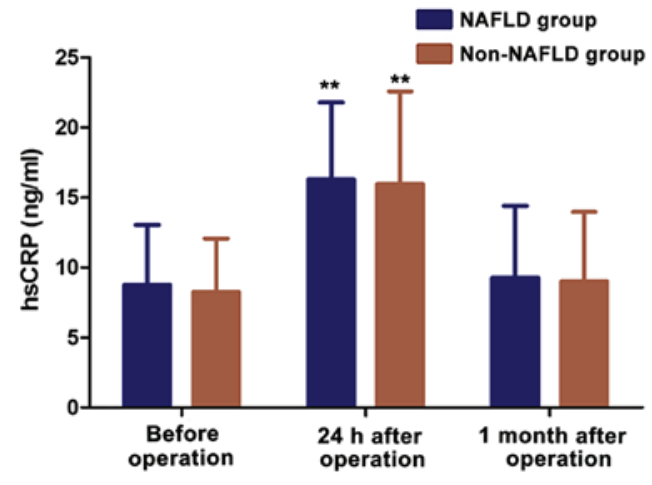

Figure 1. Expression levels of hsCRP in peripheral blood of patients in NAFLD and non-NAFLD groups at different time-points. ${ }^{* *} \mathrm{p}<0.01$ compared to levels before the procedure in the same group. hsCRP, high-sensitivity C-reactive protein; NAFLD, non-alcoholic fatty liver disease.

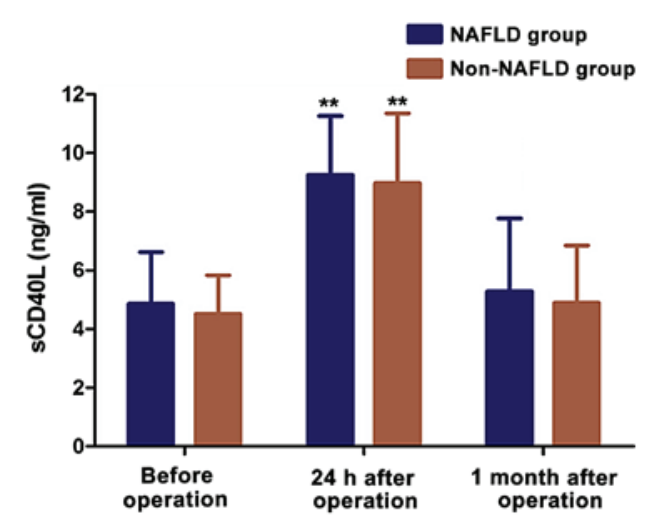

Figure 2. Expression levels of sCD40L in peripheral blood of patients in the NAFLD and non-NAFLD groups at different time-points. ${ }^{* *} \mathrm{p}<0.01$. sCD $40 \mathrm{~L}$, soluble CD40 ligand; NAFLD, non-alcoholic fatty liver disease.

Expression level of $s C D 40 \mathrm{~L}$ in peripheral blood. The expression level of sCD40L in peripheral blood of patients was detected using a SCD40L kit. The results on Table III and Fig. 2 show that the expression levels of SCD40L in NAFLD group and non-NAFLD group were highest at $24 \mathrm{~h}$ after operation in both groups $(\mathrm{p}<0.01)$. However, there were no statistically significant differences in the expression levels of sCD40L before and at one month after the operation $(p>0.05)$; and there were no statistically significant differences in the expression levels of sCD40L between the NAFLD and non-NAFLD groups ( $>0.05)$.

Expression level of ICAM-1 in peripheral blood. The expression level of ICAM-1 in peripheral blood of patients was detected using an ICAM-1 ELISA kit. The results shown on Table IV and Fig. 3 reveal a slight increase in the expression levels of ICAM-1 one day after the CABG procedure in the two groups. But there was no statistically significant difference in the expression levels between the two groups before and after the operation $(\mathrm{p}>0.05)$.

Expression level of MMP-9 in peripheral blood. The expression level of MMP-9 in peripheral blood of patients was detected using an MMP-9 kit. The results in Table V and Fig. 4 show the expression levels of MMP-9 increased significantly after 
Table V. MMP-9 expression levels in peripheral blood of patients in the two groups.

\begin{tabular}{lcc}
\hline & \multicolumn{2}{c}{ MMP-9 } \\
\cline { 2 - 3 } Detection time & $\begin{array}{c}\text { NAFLD } \\
\text { group }\end{array}$ & $\begin{array}{c}\text { Non-NAFLD } \\
\text { group }\end{array}$ \\
\hline Before operation & $17.52 \pm 3.86$ & $16.97 \pm 4.11$ \\
24 h after operation & $18.56 \pm 4.67$ & $18.22 \pm 4.35$ \\
1 month after operation & $40.18 \pm 5.51^{\mathrm{a}, \mathrm{b}}$ & $30.02 \pm 6.15^{\mathrm{a}}$ \\
\hline
\end{tabular}

Compared with non-NAFLD group at each time-point, ${ }^{\mathrm{a}} \mathrm{p}<0.01$; compared with non-NAFLD group, ${ }^{b} \mathrm{p}<0.01$. MMP-9, matrix metalloproteinase-9; NAFLD, non-alcoholic fatty liver disease.

Table VI. Percentages of adverse cardiovascular events in the two groups.

\begin{tabular}{lrcc}
$\begin{array}{l}\text { Adverse } \\
\text { cardiovascular } \\
\text { events }\end{array}$ & $\begin{array}{c}\text { NAFLD } \\
\text { group }\end{array}$ & $\begin{array}{c}\text { Non-NAFLD } \\
\text { group }\end{array}$ & P-value \\
\hline $\begin{array}{l}\text { Death (\%) } \\
\text { Angina pectoris }\end{array}$ & $3.2 \%$ & 0 & 0.356 \\
$\begin{array}{l}\text { Myocardial infarction } \\
\text { Total adverse }\end{array}$ & $6.5 \%$ & $8.1 \%$ & 0.187 \\
cardiovascular & $19.4 \%$ & $13.5 \%$ & 0.276 \\
events & & & $0.029^{\text {a }}$ \\
\hline
\end{tabular}

Compared with non-NAFLD group at each time-point, ${ }^{\mathrm{a}} \mathrm{p}<0.05$. NAFLD, non-alcoholic fatty liver disease.

the operation and reached a peak one month after the procedure $(\mathrm{p}<0.01)$. Importantly, the expression levels of MMP-9 in peripheral blood in the NAFLD group at 1 month after the $\mathrm{CABG}$ procedure were significantly higher than those in the non-NAFLD group $(\mathrm{p}<0.01)$.

Relationship between inflammatory factor levels in peripheral blood and prognosis. The number of adverse cardiovascular events was recorded for 6 months after the CABG operation through follow-up of patients. The results can be seen on Table VI. The total incidence of adverse cardiovascular events of patients in the non-NAFLD group was significantly lower than that of patients in the NAFLD group $(\mathrm{p}=0.029)$. Logistic regression analysis was used to analyze the expression levels of hsCRP, sCD40L, ICAM-1 and MMP-9 in peripheral blood of patients and the rates of adverse cardiovascular events within 6 months after operation, and the results showed that the expression level of MMP-9 was an important influencing factor of cardiovascular events after CABG $(\mathrm{OR}=1.182, \mathrm{p}<0.05)$.

\section{Discussion}

NAFLD can significantly increase the morbidity and mortality rates of coronary heart disease patients (7-9).

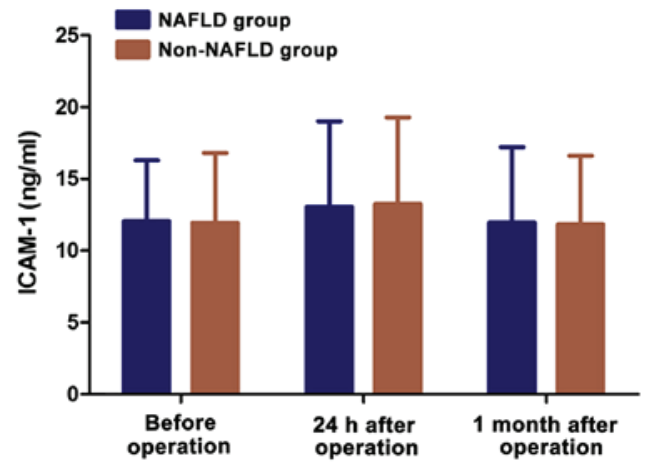

Figure 3. Expression levels of ICAM-1 in peripheral blood of patients in NAFLD and non-NAFLD groups at different time-points. ICAM-1, intercellular adhesion molecule-1; NAFLD, non-alcoholic fatty liver disease.

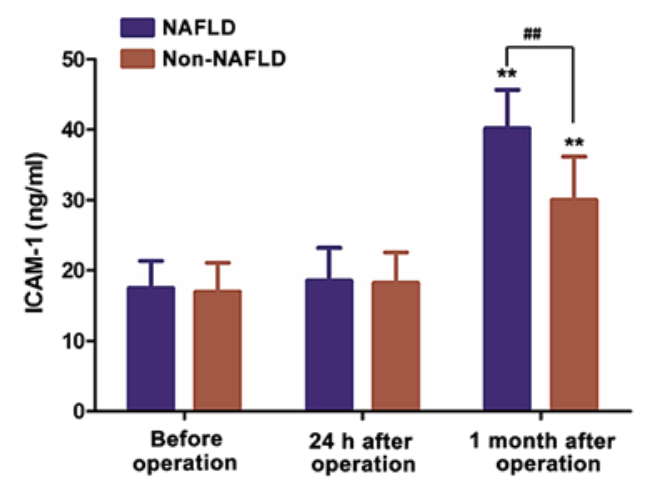

Figure 4. Expression levels of MMP-9 in peripheral blood of patients in NAFLD and non-NAFLD groups at different time-points. ${ }^{* *} \mathrm{p}<0.01$ \#"Significantly higher one month after the procedure. MMP-9, matrix metalloproteinase-9; NAFLD, non-alcoholic fatty liver disease.

The inflammatory response in vessels gets activated after bypass operation or coronary stenting, and leads to platelet aggregation and adhesion and further release of inflammatory mediators. Current studies have found that hsCRP, sCD40L, ICAM-1 and MMP-9 are commonly affected inflammatory factors (10-12). Investigating the relationship between the expression levels of inflammatory factors in peripheral blood after coronary bypass operation and the treatment outcomes in patients is important to be able to provide the patients with a more accurate prognosis.

CRP, an acute response albumin in the body, is an important factor in the inflammatory process, and is considered to be closely related to the occurrence, development and prognosis of coronary artery diseases. Studies have shown that CABG can cause vascular endothelial injury, and a series of cytokines get expressed during the process, such as CRP (13). CD40L is a surface glycoprotein that can be expressed in human atherosclerotic plaques and vascular endothelial cells (14). It was found in this study that hsCRP and SCD40L levels in peripheral blood of patients were increased $24 \mathrm{~h}$ after the CABG operation, and the levels were significantly higher than those before and at 1 month after the procedure $(\mathrm{p}<0.01)$. This is consistent with a different study where it was found that after stenting, patients with coronary artery disease had increased expression levels of hsCRP in peripheral blood (15). Likewise, Napoleão et al (16) found that the expression levels of sCD40L 
were significantly increased in peripheral blood within $10 \mathrm{~min}$ after CABG compared with those before operation, suggesting that the levels may be associated with postoperative systemic inflammatory responses. Ma et al (17) showed that the expression levels of ICAM-1 increased slightly in peripheral blood of patients in the two groups after operation, but the differences were not statistically significant. The expression level of ICAM-1 of patients in NAFLD group almost had no change. This is inconsistent with literature reports where it was found that the ICAM-1 levels were increased when using a coronary artery injury model after CABG (may be due to the vascular damage response), or that the levels were low in other conditions (may be because of a negative feedback regulation mechanisms) $(18,19)$. Then again, it was found in this study that the expression levels of MMP-9 in peripheral blood of patients in the two groups were highest one day after the CABG procedure, and that the levels in the NAFLD group were significantly higher than those in the non-NAFLD group $(\mathrm{p}<0.01)$. Furthermore, regression analysis showed that MMP-9 is an important influencing factor for adverse cardiovascular events after the bypass operation. These results are not surprising given that animal experiments had already proved that MMP-9 inhibitors can reduce restenosis after stenting, so it was suggested that MMP-9 and its inhibitors are involved in the development of in-stent restenosis (20). Based on these data, we believe that MMP-9 may be an important inflammatory mediator after coronary stenting, which is increased for a long time after the bypass operation for coronary heart disease in patients complicated with NAFLD, so it can be used as an inflammatory marker to predict adverse events of coronary heart disease in these patients.

In conclusion, the expression levels of inflammatory factors in peripheral blood of patients complicated with NAFLD undergoing $\mathrm{CABG}$ are different from those of non-complicated patients, and high levels of MMP-9 are associated with higher risk of adverse cardiovascular events after the operation. MMP-9 should be useful as an inflammatory marker for predicting the risk of adverse events for coronary heart disease patients complicated with NAFLD undergoing CABG procedures.

\section{References}

1. Sun X, Duan X, Wang C, Liu Z, Sun P, Huo X, Ma X, Sun H, Liu K and Meng Q: Protective effects of glycyrrhizic acid against non-alcoholic fatty liver disease in mice. Eur J Pharmacol 806: 75-82, 2017.

2. Yahagi $M$, Tsuruta $M$, Hasegawa $H$, Okabayashi $K$ and Kitagawa Y: Non-alcoholic fatty liver disease fibrosis score predicts hematological toxicity of chemotherapy including irinotecan for colorectal cancer. Mol Clin Oncol 6: 529-533, 2017

3. Chan WK, Nik Mustapha NR, Wong GL, Wong VW and Mahadeva S: Controlled attenuation parameter using the FibroScan ${ }^{\circledR}$ XL probe for quantification of hepatic steatosis for non-alcoholic fatty liver disease in an Asian population. United European Gastroenterol J 5: 76-85, 2017.

4. Morimoto T, Mineharu Y, Ono K and Nakatochi M: Significant association of RNF213 p.R4810K, a moyamoya susceptibility variant, with coronary artery disease. PLoS One 12: e0175649, 2017.
5. Silveira de Souza VV, Soares Torres F, Hochhegger B, Watte G, Sartori G, Lucchese F and Azambuja Gonçalves B: Performance of ultra-low-dose CT for the evaluation of coronary calcification: A direct comparison with coronary calcium score. Clin Radiol 72: 745-750, 2017.

6. Raman SV and Zareba KM: Coronary artery disease testing: Past, present, and future. JACC Cardiovasc Imaging 16: 285-297, 2017.

7. Verna EC: Non-alcoholic fatty liver disease and non-alcoholic steatohepatitis in patients with HIV. Lancet Gastroenterol Hepatol 2: 211-223, 2017.

8. Degasperi E and Colombo M: Distinctive features of hepatocellular carcinoma in non-alcoholic fatty liver disease. Lancet Gastroenterol Hepatol 2: 156-164, 2017.

9. Maurice JB, Patel A, Scott AJ, Patel K, Thursz M and Lemoine M: Prevalence and risk factors of non-alcoholic fatty liver disease in HIV-monoinfection: A systematic review and meta-analysis. AIDS 12: 487-491, 2017.

10. Sandri A, Petersen RH, Decaluwé H, Moons J, Ferguson MK, Hansen HJ and Brunelli A: Coronary artery disease is associated with an increased mortality rate following video-assisted thoracoscopic lobectomy. J Thorac Cardiovasc Surg 154: 352-357, 2017.

11. Schmidt B, Frölich S, Dragano N, Frank M, Eisele L, Pechlivanis S, Forstner AJ, Nöthen MM, Mahabadi AA, Erbel R, et al: Socioeconomic status interacts with the genetic effect of a chromosome $9 \mathrm{p} 21.3$ common variant to influence coronary artery calcification and incident coronary events in the Heinz Nixdorf Recall study (risk factors, evaluation of coronary calcium, and lifestyle). Circ Cardiovasc Genet 10: e001441, 2017.

12. Ebell MH: PCI has more benefits and harms than CABG for selected patients with left main coronary artery disease. Am Fam Physician 95: 455-456, 2017.

13. Kodama R, Muraki S, Iidaka T, Oka H, Teraguchi M, Kagotani R, Asai Y, Hashizume H, Yoshida M, Kawaguchi H, et al: Serum levels of matrix metalloproteinase-3 and autoantibodies related to rheumatoid arthritis in the general Japanese population and their association with osteoporosis and osteoarthritis: The ROAD study. J Bone Miner Metab 6: 435-447, 2017.

14. Gudbrandsdottir S, Ghanima W, Nielsen $\mathrm{CH}$, Feng X, Hasselbalch HC and Bussel J: Effect of thrombopoietin-receptor agonists on circulating cytokine and chemokine levels in patients with primary immune thrombocytopenia (ITP). Platelets 28 : 478-483, 2017.

15. Yoo HJ, Hwang SY, Choi JH, Lee HJ, Chung HS, Seo JA, Kim SG, Kim NH, Baik SH, Choi DS, et al: Association of leukocyte cell-derived chemotaxin 2 (LECT2) with NAFLD, metabolic syndrome, and atherosclerosis. PLoS One 12: e0174717, 2017.

16. Napoleão P, Potapova E and Moleirinho S: Soluble CD40 ligand profiles in patients with septic shock. Clin Hemorheol Microcirc 9: 25-32, 2014.

17. Ma S, Chen X, Wang L and Wei Y: Repairing effects of ICAM-1-expressing mesenchymal stem cells in mice with autoimmune thyroiditis. Exp Ther Med 7: 25-32, 2012.

18. Abo-Hagar HH, Abo-Elezz AA, Mehrez M, Mabrouk MM and Elshora OA: Diagnostic efficacy of serum amyloid A protein and soluble intercellular adhesion molecule 1 in pediatric ventilator-associated pneumonia. J Intensive Care Med 83: 885066617702598, 2017. doi: 10.1177/0885066617702598.

19. Walker DG, Lue LF, Tang TM and Adler CH: Changes in CD200 and intercellular adhesion molecule-1 (ICAM-1) levels in brains of Lewy body disorder cases are associated with amounts of Alzheimer's pathology not $\alpha$-synuclein pathology. Neurobiol Aging 15: 97-110, 2013

20. Mahmoodi K, Kamali K and Karami E: Plasma concentration, genetic variation, and gene expression levels of matrix metalloproteinase 9 in Iranian patients with coronary artery disease. J Res Med Sci 4: 328-335, 2010.

This work is licensed under a Creative Commons Attribution-NonCommercial-NoDerivatives 4.0 International (CC BY-NC-ND 4.0) License. 